\title{
Title:
}

\section{Global Mapping at the Core of the Arabidopsis Circadian Clock Defines the Network Structure of the Oscillator}

\author{
Authors: W. Huang ${ }^{1}$, P. Pérez-García ${ }^{1}$, A. Pokhilko ${ }^{2}$, A.J. Millar ${ }^{2,3}$, I. Antoshechkin ${ }^{4}$, J.L. \\ Riechmann ${ }^{1,4,5}$ and P. Mas ${ }^{1 *}$
}

\author{
Affiliations: \\ ${ }^{1}$ Center for Research in Agricultural Genomics (CRAG), Barcelona 08193, Spain. \\ ${ }^{2}$ School of Biological Sciences, University of Edinburgh, Edinburgh EH9 3JH, United \\ Kingdom. \\ ${ }^{3}$ Centre for Systems Biology at Edinburgh, C.H. Edinburgh EH9 3JD, United Kingdom. \\ ${ }^{4}$ California Institute of Technology, Division of Biology, Pasadena, CA 91125, USA. \\ ${ }^{5}$ Institució Catalana de Recerca i Estudis Avançats, Barcelona 08010, Spain. \\ *Correspondence to: paloma.mas@cragenomica.es
}

\begin{abstract}
:
In many organisms, the circadian clock is composed of functionally coupled morning and evening oscillators. In Arabidopsis, oscillator coupling relies on a core loop in which the evening oscillator component TOC1 (TIMING OF CAB EXPRESSION1) was proposed to activate a subset of morning-expressed oscillator genes. Here we show that TOC1 does not function as an activator but as a general repressor of oscillator gene expression. Repression occurs through TOC1 rhythmic association to the promoters of the oscillator genes. Hormone-dependent induction of TOC1 and analysis of RNAi plants show that TOC1 prevents the activation of morning-expressed genes at night. Our study overturns the prevailing model of the Arabidopsis circadian clock showing that the morning and evening oscillator loops are connected through the repressing activity of TOC1.
\end{abstract}

One Sentence Summary: The morning and evening feedback loops at the core of the Arabidopsis oscillator are connected through the repressing activity of the key clock component TOC1. 


\section{Main Text:}

The molecular circuitry underlying circadian rhythms relies on the reciprocal regulation of clock components forming negative feedback loops at the core of the oscillator (1). However, deciphering the oscillator transcriptional regulatory code is a major challenge due to the interplay among clock activators and repressors, which are responsible for the generation of the loops. In Arabidopsis, two MYB transcription factors, CIRCADIAN CLOCK ASSOCIATED 1 (CCA1) and LATE ELONGATED HYPOCOTYL (LHY) $(2,3)$ together with the pseudo-response regulators PRR5, 7 and 9 (4) function as key components of a so-called morning loop, which interlocks with an evening loop $(5,6)$ composed of TOC1 (or PRR1) (4, 7), GIGANTEA (GI) (8, 9) and a hypothetical component Y (10). The evening loop also includes the Evening Complex (EC) composed of EARLY FLOWERING 3 (ELF3), 4 (ELF4) and LUX ARRHYTHMO (LUX) $(11,12)$.

Regulation of TOC1 rhythmic expression is essential for proper functioning of the clock $(13,14)$. Mechanisms that contribute to this regulation include changes in chromatin structure (15), transcriptional regulation (16) and protein degradation (17). Alterations of this regulation affect clock function and result in disrupted circadian gene expression and changes in time to flower $(7,18,19)$. TOC1 also connects environmental signals with clock-controlled photomorphogenic and hormonal outputs $(18,20)$. Gene expression analysis using toc1 mutant plants led to the hypothesis that TOC1 inhibits its expression by activating the repressors CCA1 and LHY $(16,21)$. The direct interaction of TOC1 with CCA1 HIKING EXPEDITION (CHE) was proposed as a regulatory link connecting TOC1 with the activation of CCA1 (21). Our study changes the sign of TOC1 function at the core of the clock and shows that the morning and evening feedback loops are connected through the repressing activity of TOC1.

We used chromatin immunoprecipitation followed by deep sequencing (ChIP-Seq) to obtain a high-resolution map of TOC1 chromatin occupancy on a genome-wide scale (22). The ChIP experiments were performed with TOC1 Minigene (TMG) seedlings expressing the genomic fragment of TOC1 fused to the Yellow Fluorescent Protein (18) in the toc1-2 (hypomorphic mutant) background (7) (fig. S1). ChIP-Seq data analysis in search for genes that contained one or more TOC1 binding regions identified 867 peak-to-gene associations (ChIPSeq peak p-value $\leq 0.001$ ) corresponding to 772 potential TOC1 target genes (Supporting Online Material, table S1 and fig. S2). Consistent with previous studies $(20,21)$, we found that CCA1 
and $A B A R / C H L H / G U N 5$, binding targets of TOC1, were present in our gene list. The $A B A R$ circadian expression is repressed by TOC1 (20).

As TOC1 is a key component of the Arabidopsis circadian system, we analyzed the proportion of TOC1-binding genes that were under the control of the circadian clock. Using different circadian gene expression datasets $(23,24)$, we found that $40 \%$ of TOC1 targets were regulated by the circadian clock (table S1, S2, S6 and fig. S3, S4). Analysis of peak phases of expression revealed a phase enrichment around dawn (Fig. 1A-C, table S3), which suggests that TOC1 modulates clock genes expressed during the morning phase. We also found that the promoters of the oscillator components were occupied by TOC1. The targets included genes from both the morning (CCA1, LHY, PRR9, PRR7) and the evening loops (GI, TOC1, ELF4 and $L U X)$ (Fig. 1D-G and table S4). We also identified other components associated to the oscillator such as PRR5 (13) whereas other clock-related genes more indirectly linked to the transcriptional regulation of the oscillator such as ZTL, FKF1 or LKP2 (13) or clock genes expressed only in specific tissues (e.g. PRR3) (13) were not found as putative TOC1 targets. Independent ChIP-QPCR analyses showed specific amplification of the regions identified by ChIP-Seq, consistent with the binding of TOC1 to the promoters of the oscillator genes (fig. S5). We further validated our results by using TOC1 over-expressing plants (TOC1-ox), in which TOC1 is constitutively expressed. In these plants, TOC1 occupancy at the oscillator loci was significantly higher than occupancy at the promoters of genes not present in our ChIP-Seq list (fig. S5). We therefore report the association of TOC 1 to the promoters of the morning and evening oscillator genes. Binding might be indirect and may require other co-factors and/or the formation of higher order protein complexes.

Analysis of TOC1-bound oscillator sequences identified as significantly enriched a motif closely related to the GBox (named GBox-expanded) (Fig. 1H, fig. S6 and table S5). We also found that a motif very similar to the circadian motif, Evening Element (EE), was present in both morning and evening-expressed oscillator genes (named EE-like-expanded) (Fig. 1I, fig. S6). The EE motif was proposed to be associated with evening-expressed genes $(16,25)$. The motifs were positioned very close to the center of the TOC1-bound ChIP-Seq genomic regions (fig. S6). A similar positional enrichment at the peak maximum was observed when sequences of the circadian genes bound by TOC1 were analyzed (fig. S6). The results were consistent with a 
ChIP-Q-PCR screening of the CCA1 promoter, showing that amplicon enrichment decreased as the distance from the GBox and EE-like motifs increased (fig. S6).

We next analyzed the role of the circadian clock in modulating TOC1 interaction with its target genes by performing ChIP assays with TMG plants synchronized under $12 \mathrm{~h}$ light:12h dark cycles (LD). The results revealed a rhythmic oscillation of TOC1 binding with a peak at Zeitgeber Time 15 (ZT15 - 3 hours after lights off; ZT0: lights on), the proposed time of TOC1 function (Fig. 2A-D). Similar oscillatory binding was observed in plants grown under constant light (LL) conditions (Fig. 2E-F) indicating a role for the circadian clock. The rhythmicity was abolished in TOC1-ox plants (Fig. 2G-H), suggesting that TOC1 binding is dictated by its protein abundance, which is under circadian regulation. These results together with the arrhythmic phenotypes of TOC1-ox (18) support the notion that the circadian pattern of TOC1 binding is relevant for TOC1 function in the clock.

We next examined oscillator gene expression in WT and TOC1-ox samples under LD and LL cycles. The peak expression of transcripts from the morning loop genes (PRR9, PRR7, CCA1 and LHY) was significantly reduced in TOC1-ox (Fig. 2I-J, fig. S7, table S7). This is noteworthy as TOC1 was previously assumed to be an activator of CCA1 and LHY expression (16). The reduced expression cannot be attributed to the activation of their known repressors PRR7 and PRR9, as their expression is also decreased in TOC1-ox plants. Analysis of the evening loop also revealed gene repression. For instance, GI and ELF4 were reduced around dusk (Fig. 2K-L) and plants expressing the LUX promoter fused to the LUCIFERASE (LUX::LUC) also displayed a reduced promoter activity particularly during the night (fig. S8). In the morning loop, oscillator components that are expressed at a similar phase repress each other expression (e.g. CCA1 and LHY) $(2,3)$. Our results suggest that similar regulation occurs in the evening loop, where TOC1 might also repress genes expressed during the night. The observed function of TOC1 as a repressor is not likely due to constitutive over-expression of TOC 1 as we also observed decreased oscillator expression in TMG plants (expressing TOC1 under its own promoter) (Fig. 2I-L).

To further confirm the transcriptional repressing function of TOC1, we generated transgenic plants expressing TOC1 fused to the GR domain of the glucocorticoid receptor (26). In the absence of a steroid ligand, the GR domain retains a nuclear factor in the cytoplasm but 
nuclear localization is restored in the presence of the synthetic glucocorticoid Dexamethasone (Dex). Treatment of TOC1-GR plants with Dex resulted in a reduction of CCA1 and GI expression relative to the mock-treated controls (Fig. 3A-B and fig. S9). Our analysis revealed statistically significant differences between TOC1-GR+Dex and the other genotypes (+/-Dex) (Fig. 3D-E) while no significant variations were found in WT plants in the presence or in the absence of Dex. We also analyzed the effects of TOC1-GR induction by Dex on CCA1::LUC activity. The in vivo studies were consistent with the RT-Q-PCR data and showed a clear reduction of CCA1::LUC luminescence in Dex treated plants (Fig. 3C). To further address the repressing function of TOC1, we examined CCA1 expression in TOC1-GR plants treated with Dex in the presence or in the absence of the protein synthesis inhibitor Cycloheximide (CHX). Our results showed that CCA1 expression was reduced after Dex treatment in the presence of CHX (Fig. 3F) indicating that CCA1 repression does not require de novo protein synthesis. We also utilized the previously described induction of TOC1 by the plant hormone abscisic acid (ABA) (20). We analyzed TOC1 induction by ABA and in parallel we examined CCA1 and PRR9 expression. The results showed that the induction of TOC1 was accompanied by a concomitant reduction of CCA1 and PRR9 expression (Fig. 3G-I). Our studies, together with the results of toc1 mutant plants (see Fig. 4) indicate that in contrast to its previously assigned activating role, TOC1 functions as a repressor of oscillator gene expression.

The repressive function of TOC1 provides an explanation for recently published experimental data that cannot be reconciled with an activating role for TOC1. For instance, analysis of ztl mutant plants, with increased TOC1 accumulation (17), revealed a reduced expression of LHY and CCA1 (27), which is not consistent with TOC1 activating function. The data also showed low abundance of PRR9 and PRR7 expression (27). Thus, the reduced expression of $L H Y$ and CCA1 cannot be attributed to increased abundance of their inhibitors. To clarify TOC1 function in the clock, we examined oscillator gene expression in TOC1 RNAi and toc1-2 mutant plants grown under LD cycles. Our results showed an advanced phase for $L H Y$ expression at the onset of transcription (Fig. 4A and fig. S10) suggesting that the absence of TOC1 alleviates the repression normally observed in WT plants around dusk. The advanced phase of $L H Y$ expression correlated with a slight increase of PRR7 (Fig. 4B and fig. S10). These results suggest that TOC1 temporally extends the repressing function of the other sequentially expressed PRRs inhibitors (28) (Fig. 4E). The absence of TOC1 in toc1 mutant plants shortens 
the duration of this repression and as a consequence, the onset of $L H Y$ transcription is advanced (Fig. 4F). The effects on the trough of $L H Y$ are subtle, most likely because the expression of other CCA1/LHY repressors is also increased (Fig. 4C-D). In TOC1-ox, the abnormally high expression of TOC1 during the day reveals its repressing function at times when TOC1 is not normally expressed (Fig. 4G). Altogether, our studies support the repressing function of TOC1 on oscillator gene expression, which changes the regulatory circuit at the core of the Arabidopsis circadian clock (fig. S11).

Conclusions about the circadian transcriptional networks are complicated by the existence of multiple feedback loops and gene redundancies. Our study shows the complex and dynamic function of TOC1 as a regulator of oscillator gene expression, with a repressing activity of the morning and evening loops. Binding of TOC1 to the promoters of the oscillator genes may antagonize transcriptional activation around the time of peak expression. The mechanisms of oscillator gene activation are largely unknown but chromatin remodelling and light-dependent regulation may play key roles at defined times of day. The challenge remains now to identify the mechanisms and components responsible for the activation of oscillator gene expression.

\section{References and Notes}

1. H. Wijnen, M. W. Young, Annu. Rev. Genet. 40, 409 (2006).

2. $\quad$ R. Schaffer et al., Cell 93, 1219 (1998).

3. Z. Y. Wang, E. M. Tobin, Cell 93, 1207 (1998).

4. A. Matsushika, S. Makino, M. Kojima, T. Mizuno, Plant Cell Physiol. 41, 1002 (2000).

5. J. C. Locke et al., Mol. Syst. Biol. 1, 1 (2005).

6. M. N. Zeilinger, E. M. Farre, S. R. Taylor, S. A. Kay, F. J. Doyle, Mol. Syst. Biol. 2, 1 (2006).

7. C. A. Strayer et al., Science $\mathbf{2 8 9}, 768$ (2000).

8. $\quad$ S. Fowler et al., EMBO J. 18, 4679 (Sep 1, 1999).

9. D. H. Park et al., Science 285, 1579 (1999).

10. A. Pokhilko et al., Mol Syst Biol. 6, 1 (2010).

11. D. A. Nusinow et al., Nature 475, 398 (2011).

12. E. Herrero et al., Plant Cell, doi: 10.1105/tpc.111.093807 (2012).

13. C. R. McClung, R. A. Gutiérrez, Curr Opin Genet Dev 20, 588 (2010).

14. P. Más, Trends Cell Biol. 18, 273 (2008).

15. M. Perales, P. Más, Plant Cell 19, 2111 (2007).

16. D. Alabadí et al., Science 293, 880 (2001).

17. P. Más, W. J. Kim, D. E. Somers, S. A. Kay, Nature 426, 567 (2003).

18. P. Más, D. Alabadí, M. J. Yanovsky, T. Oyama, S. A. Kay, Plant Cell 15, 223 (2003).

19. D. E. Somers, A. A. R. Webb, M. Pearson, S. A. Kay, Development 125, 485 (1998).

20. T. Legnaioli, J. Cuevas, P. Mas, EMBO J 28, 3745 (2009). 
21. J. L. Pruneda-Paz, G. Breton, A. Para, S. A. Kay, Science 323, 1481 (2009).

22. Materials and methods are available as supporting material on Science Online.

23. M. Covington, J. Maloof, M. Straume, S. A. Kay, S. Harmer, Genome Biol. 9, R130 (2008).

24. S. P. Hazen et al., Genome Biol. 10, R17 (2009).

25. T. P. Michael et al., PLoS Genetics 4, e14 (2008).

26. A. Lloyd, M. Schena, V. Walbot, R. Davis, Science 266, 436 (1994).

27. A. Baudry et al., The Plant Cell 22, 606 (2010).

28. $\quad$ N. Nakamichi et al., The Plant Cell 22, 594 (2010).

29. S. J. Clough, A. F. Bent, Plant J. 16, 735 (1998).

30. J. C. Carrington, D. D. Freed, A. J. Leinicke, Plant Cell. 3, 953 (1991).

31. P. Hajdukiewicz, Z. Svab, P. Maliga, Plant Mol Biol. 25, 989 (1994).

32. P. A. Salomé, C. R. McClung, Plant Cell 17, 791 (2005).

33. S. Portolés, P. Más, PLoS Genet 6, e1001201 (2010).

34. J. D. Plautz et al., J. Biol. Rhythms 12, 204 (1997).

35. S. Pepke, B. Wold, A. Mortazavi, Nat Meth 6, S22 (2009).

36. J. W. Nicol, G. A. Helt, S. G. Blanchard, A. Raja, A. E. Loraine, Bioinformatics 25, 2730 (2009).

37. T. Mockler et al., Cold Spring Harb Symp Quant Biol 72, 353 ( 2007).

38. J. M. Carlson, A. Chakravarty, C. E. DeZiel, R. H. Gross, Nucleic Acids Research 35, W259 (2007).

39. A. Chakravarty, J. Carlson, R. Khetani, R. Gross, BMC Bioinformatics 8, 249 (2007).

40. J. M. Carlson, A. Chakravarty, R. H. Gross, Journal of Computational Biology 13, 686 (2006).

41. J. Carlson, A. Chakravarty, R. Khetani, R. Gross, BMC Bioinformatics 7, 254 (2006).

42. A. Chakravarty, J. M. Carlson, R. S. Khetani, C. E. DeZiel, R. H. Gross, Bioinformatics 23, 1029 (2007).

43. S. P. Hazen et al., Proc. Natl. Acad. Sci. USA 102, 10387 (2005).

44. A. J. Millar, I. A. Carre, C. A. Strayer, N. H. Chua, S. A. Kay, Science 267, 1161 (1995).

45. K. Onai, M. Ishiura, Genes Cells 10, 963 (2005).

\section{Acknowledgments:}

We thank Dr. Elliot Meyerowitz for comments on the manuscript; E. Centeno for help with ChIP-Seq analysis; O. Casagran for help with the microfluidic arrays; M. Perales for help with the ChIP and L. Rico for help with the inducible constructs; L. Schaeffer and D. Trout (Jacobs Genetics and Genomics Laboratory at Caltech) for the primary sequence data processing. This work was supported by grants to P.M. from the Ramón Areces Foundation, the Spanish Ministry of Science and Innovation (MICINN), the EMBO YIP program and from the European Heads of Research Councils and the European Science Foundation through the EURYI Award; and to J.L.R. from the EC Marie Curie program and MICINN; and by the European Commission FP7 Collaborative Project TiMet to A.J.M. and others. The Centre for Systems Biology at 
Edinburgh is a Centre for Integrative and Systems Biology supported by BBSRC and EPSRC award D019621. W.H. is supported by a Juan de la Cierva contract (MICINN) and P.P.G. by a FPI fellowship (MICINN). Sequencing data have been deposited with the National Center for Biotechnology Information Gene Expression Omnibus under accession number GSE35952. 
Fig. 1. Analysis of TOC1 ChIP-Seq circadian targets. Global trend of expression phases within TOC1 circadian targets $(\mathbf{A}, \mathbf{B})$ and for a random list of circadian genes $(\mathbf{C})$. Phase enrichments were graphically portrayed within a range of ChIP-Seq p-values (legends) at different Zeitgeber Times (radial axis: 0-dawn; 12-dusk). The white and grey areas represent day and night, respectively. Peak traces of $L H Y(\mathbf{D})$, PRR7 (E) PRR9 (F) and GI (G) from TOC1 ChIP-Seq data. The exon-intron-UTR structure of the different gene models above and below each panel indicates forward and reverse orientation, respectively. Position weight matrix representation of consensus motifs present within the TOC 1 target oscillator sequences. The Gbox-expanded $(\mathbf{H})$ and EE-like-expanded (I) motifs were identified using the SCOPE motif finder. Both strands were used to compute the significance value. ChIP-Seq, peak visualization, circadian phase analysis and motif discovery were performed as described in Supporting Online Material.

Fig. 2. TOC1 binds to the promoters of the oscillator genes and inhibits their expression. ChIPQ-PCR assays of TMG plants sampled every four hours over a 24-hour LD cycle. Primers encompassing target sequences obtained in the ChIP-Seq analysis were used to amplify CCA1 and LHY (A); PRR7 and PRR9 (B), GI and PRR5 (C) or LUX/PCL1 and ELF4 (D). Binding was also examined under LL conditions following entrainment in TMG (E-F) and in TOC1-ox plants (G-H). Values are represented as means \pm SEM. Expression profiling of PRR9 (I), PRR7 (J), GI (K) and ELF4 (L) in WT, TMG and TOC1-ox plants. Gene expression was analyzed in plants grown under LD cycles. mRNA abundance was normalized to IPP2 expression. Values represent means \pm SEM. White boxes: day; grey boxes: night.

Fig. 3. Effects of TOC1 transient induction on oscillator gene expression. Analysis of CCA1 (A) and GI (B) in WT and TOC1-GR plants mock-treated or treated with $5 \mu \mathrm{M}$ dexamethasone (+Dex). Seedlings were treated at ZT10 and samples were analyzed at the indicated ZT. (C) Analysis of CCA1::LUC luminescence in TOC1-GR seedlings under $8 \mathrm{~h}$ light:16h dark cycles. Luminescence was recorded 24 hours after the seedlings were transferred to medium containing $5 \mu \mathrm{M}$ of Dex. Data are presented as means \pm SEM of luminescence signals from 6-7 independent plants. (D, E and F) Comparisons of CCA1 expression in TOC1-GR plants mock-treated or treated with $5 \mu \mathrm{M}$ of Dex at ZT10 and analyzed at ZT21 and ZT23 in the absence (-CHX) or in the presence $(+\mathrm{CHX})$ of $50 \mu \mathrm{M}$ of cycloheximide. The statistical relevance of the differences is presented (*p-value $<0.05 ; * *$ p-value $<0.01 ; * * *$ p-value $<0.001)$. (G, $\mathbf{H}$ and I) Time course analysis of TOC1, CCA1 and PRR9 expression over the diurnal cycle in TMG plants in the presence or in the absence of the hormone Abscisic Acid (ABA). Seedlings were sprayed with $100 \mu \mathrm{M}( \pm)$ ABA at ZT5. The Y axes of the CCA1 and PRR9 graphs were divided into segments so that repression at trough can be clearly observed. White boxes: day; grey boxes: night.

Fig. 4. Gene expression analysis in TOC1 RNAi plants. Analysis of $L H Y$ (A) and PRR7 (B) expression under 12L:12D conditions. Gene expression analysis of PRR9 (C) and GI (D) during the night in TOC1 RNAi under 12L:12D conditions. Values are represented as means \pm SEM. Analysis was performed as described in Supporting Online Material. Schematic diagrams depicting the waveform of CCA1/LHY expression and the different waves of PRRs repression. Schemes depict the regulations in WT (black line) (E), toc1 mutant (blue line) (F) and TOC1-ox (red line) (G). The small lines ending in perpendicular dashes represent repression. White boxes: day; black boxes: night. 


\section{Supporting Online Material}

Materials and Methods

SOM Text

Figures S1-S1 1

Tables S1-S7

References (29-45) 